Investigaciones Fenomenológicas, n. 9, 2012, 157-172.

e-ISSN: $1885-1088$

\title{
EXPERIENCIA Y "A-FILOSOFÍA": UNA APROXIMACIÓN A LA LeCtuRA Merleau-Pontyana del Pensamiento de Hegel
}

\author{
Maximiliano B. Cladakis \\ UNSAM-CONICET, Argentina \\ maxicladakis@yahoo.com.ar
}

\begin{abstract}
Resumen: El objetivo del siguiente trabajo es abordar la forma en que Merleau-Ponty ve en la filosofía expuesta por Hegel en la Fenomenología del espíritu una modalidad de pensamiento que se abre a la experiencia histórica del hombre en toda su riqueza y complejidad. Con esta finalidad, nuestra exposición se centrará en la nota de curso "Filosofía y no-filosofía después de Hegel", texto en el cual el pensamiento de Hegel es definido como "a-filosofía". En este sentido, nos interesa destacar que la lectura que Merleau-Ponty realiza de Hegel se encuentra motivada por la preocupación de poder pensar una experiencia que excluya toda posición de sobrevuelo (surplomb) y toda separación entre la conciencia y el objeto.
\end{abstract}

Palabras clave: Merleau-Ponty, fenomenología, Hegel, experiencia, dialéctica.

\begin{abstract}
The aim of this paper is to address how Merleau-Ponty sees in philosophy expounded by Hegel in the "Phenomenology of Spirit" a mode of thinking that opens to the historical experience of man in all its richness and complexity. With this purpose, our discussion will focus on the course mark "Philosophy and non-philosophy after Hegel" text in which Hegel's thought is defined as "aphilosophy". In this regard, we wish to emphasize that Merleau-Ponty makes a Hegel reading motivated by a concern to think that experience excludes any overflight position (surplomb) and any separation between consciousness and object.
\end{abstract}

Keywords: Merleau-Ponty, phenomenology, experience, dialectic.

\section{INTRODUCCIÓN}

La lectura e interpretación del pensamiento hegeliano ha sido uno de los temas que ha atravesado el desarrollo de la obra de Merleau-Ponty desde los tempranos tiempos de La estructura del comportamiento hasta los escritos publicados de manera póstuma. En este sentido, las ideas y los debates en torno al filósofo alemán han ejercido una influencia destacada sobre el autor de la Fenomenología de la percepción. Si bien dicha influencia tal vez no haya tenido una impronta tan significativa como la de Husserl o, en menor medida, Heidegger, se trata, de todas formas, de un fenómeno cuya importancia es relevante.

En su ya clásica obra Lo mismo y lo otro, Vincent Descombes considera a Merleau-Ponty, junto a Sartre, como uno de los más importantes representantes de aquella generación de intelectuales franceses que fue marcada por el 
pensamiento hegeliano, sobre todo, por la lectura llevada a cabo por Kojève en los cursos dictados entre 1933-1939¹. Más allá de las críticas que pueden realizarse a algunas de las tesis que Descombes presenta en su obra, la importancia que Merleau-Ponty le otorga a Hegel es manifestada de manera explícita en más de una ocasión. Muy probablemente, la más expresiva de ellas pueda encontrarse en el artículo "El existencialismo de Hegel", publicado en Sentido y sinsentido, donde el propio Merleau-Ponty afirma que "Hegel está en el origen de todo lo importante que se ha hecho en filosofía desde hace un siglo [... $]^{\prime 2}$.

Precisamente, nuestro objetivo, en las siguientes páginas, será abordar la forma en que Merleau-Ponty ve en la filosofía de Hegel una modalidad de pensamiento que se abre a la experiencia histórica del hombre y que, por tanto, no reduce la existencia humana al ámbito de la reflexión. Para ello, nos centraremos, principalmente, en la nota de curso "Filosofía y no-filosofía después de Hegel" $^{3}$, texto en el cual el pensamiento de Hegel es definido como "a-filosofía". En este escrito, la lectura que Merleau-Ponty realiza de Hegel, más precisamente de la Fenomenología del espíritu, se encuentra motivada por la preocupación de poder pensar una experiencia que excluya toda posición de sobrevuelo (surplomb) y toda separación entre la conciencia y el objeto. Merleau-Ponty encontrará en la obra del filósofo alemán una modalidad de pensamiento en donde sólo la experiencia se devela como la única vía capaz de dar acceso a la dialéctica. Nuestra intención es, por tanto, destacar la forma en que el fenomenólogo francés lee a Hegel a partir de su propio pensamiento y lo ve, en cierta medida, como un precursor de varias de sus propias propuestas, aunque eso no le imposibilite realizar críticas a varios supuestos del pensamiento hegeliano.

Nuestro trabajo, a su vez, estará articulado en cuatro puntos. El primero girará en torno a los conceptos de "filosofía" y de "no-filosofía" en la obra de Merleau-Ponty y al lugar que ocupa el pensamiento de Hegel dentro de esta problemática. El segundo, se centrará sobre la lectura que Merleau-Ponty realiza de la forma en que, para Hegel, se da la relación entre saber y absoluto. En el tercer punto, nos dedicaremos a abordar el rol de la negatividad y de la críti-

\footnotetext{
${ }^{1}$ Vicent Descombes, Lo mismo y lo otro. Cuarenta y cinco años de filosofía francesa (1933-1978), Madrid, Cátedra, 1979.

${ }^{2}$ Maurice Merleau-Ponty, Sens et non-sens, Paris, Nagel, 1948, p. 109.

${ }^{3}$ Maurice Merleau-Ponty, Notes de cours au Collège de France 1958-1959 et 1960-1961, Paris, Gallimard, 1996.
} 
ca en la exposición hegeliana según Merleau-Ponty. El cuarto y último punto tendrá como objetivo señalar la forma en que Merleau-Ponty comprende a la dialéctica hegeliana como dialéctica de la ambigüedad.

\section{FILOSOFÍA Y "A-FILOSOFÍA"}

En el desarrollo de su obra, Merleau-Ponty ha problematizado de manera recurrente el concepto de "filosofía". Si bien dicha problematización no se ha consolidado en un trabajo sistemático, sino que se ha tratado esencialmente de esbozos fragmentarios, muchas veces ambiguos y no exentos de contradicciones entre sí, puede encontrarse, no obstante, una línea de continuidad que subyace a los diversos textos en los que el tema es trabajado. En este sentido, al avanzar en la lectura de sus obras, no es difícil observar que, en MerleauPonty, existe un intento constante por alcanzar una forma de pensamiento que no se limite al ámbito de la reflexión o de la especulación teórica, sino que, por el contrario, se encuentre arraigada en la vida, en la historia y en la experiencia concreta del hombre en el mundo.

En este sentido, Merleau-Ponty hablará varias veces, sobre todo en sus trabajos posteriores a 1955, de la oposición entre filosofía y no-filosofía. Sin embargo, la caracterización de estos dos polos de oposición será presentada de manera ambigua y fragmentaria, variando de acuerdo al texto del cual se trate. En la nota de trabajo "Origen de la verdad" ${ }^{4}$, por ejemplo, el concepto de "nofilosofía" es empleado en un contexto de alarma para referirse a la ausencia de racionalidad y de fundamentos de la época contemporánea, mientras que en "Nuestra edad de no-filosofía" el término aparece asociado al arte y al psicoanálisis ${ }^{5}$.

Dentro de esta problemática, la presencia de Hegel, a veces implícita, otras veces explícita, suele ser frecuente, e, incluso, en algunos escritos la obra hegeliana llega a ser presentada como instancia de ruptura en el desarrollo del pensamiento occidental. En el resumen de curso "Posibilidad de la filosofía", Merleau-Ponty afirma que, luego de Hegel, se ha abierto una era de vacío fi-

\footnotetext{
${ }^{4}$ Maurice Merleau-Ponty, Le visible et I'invisible, Paris, Gallimard, 1964, p 219.

${ }^{5}$ Maurice Merleau-Ponty, Notes de cours au Collège de France 1958-1959 et 1960-1961, p. 39.
} 
losófico que aún hoy no ha podido ser superado. "Hay después de Hegel un vacío filosófico, lo que no quiere decir que hayan faltado pensadores o genios, sino que Marx, Kierkegaard y Nietzsche comienzan por una denegación de la filosofía" $^{\prime 6}$. En este sentido, Merleau-Ponty observa que, con estos autores, se entra en una edad de no-filosofía. Para Merleau-Ponty, existe una fuerte relación entre este vacío filosófico y la problemática del mundo contemporáneo. En el caso de Marx y de Nietzsche, sostiene que sus obras exponen, cada una a su manera, la problemática del mundo contemporáneo de manera muy acertada. Sin embargo, no ocurre lo mismo con las respuestas que intentaron ya que ni el proletariado como clase universal ni la voluntad de poder constituyeron una superación de la crisis.

En "Filosofía y no-filosofía después de Hegel", Merleau-Ponty también habla de la no-filosofía como una negación de la filosofía. Sin embargo, a diferencia del texto anterior, en esta nota de curso se encarga de precisar una forma de no-filosofía que no sólo niega la filosofía, sino que la realiza. Merleau-Ponty la denomina "a-filosofía". La "a-filosofía" aparece definida como un pensamiento que es, esencialmente, "[...] filosofía negativa, en el sentido de "'teología negativa', que se abre acceso a lo absoluto, no como más allá, segundo un orden positivo, sino como otro orden que exige el más acá, el doble, que solo es accesible a través de él"7. La "a-filosofía", entonces, se presenta como un pensar abierto al mundo, en una relación intrínseca con la historia y con la experiencia concreta. En esa instancia, la "a-filosofía" es la verdadera filosofía en tanto se realiza como filosofía al negarse como tal ${ }^{8}$.

Como el título mismo del escrito lo indica, en esta nota de curso Hegel ocupa un lugar fundamental. Sin embargo, respecto a las afirmaciones vertidas en "Posibilidad de la filosofía" se advierte una diferencia: en esta ocasión, Hegel aparece junto a Marx, Nietzsche y Kierkegaard como representante de esta modalidad de "no-filosofía" que es la "a-filosofía". En este aspecto, MerleauPonty realiza una crítica de las lecturas "escolarizadas", que hacen de Hegel el fundador de un sistema metafísico y sostiene que, incluso, la reacción contra la

\footnotetext{
142.

${ }^{6}$ Maurice Merleau-Ponty, Résumés de cours. Collège de France 1952-1960, Paris, Gallimard, 1968, p.

${ }^{7}$ Maurice Merleau-Ponty, Notes des cours au Collège de France 1958-1959 et 1960-1961, p. 275.

${ }^{8}$ En este punto es interesante destacar que ya en la Fenomenología de la percepción, Merleau-Ponty sostiene: "Trátese de cosas o de situaciones históricas, la filosofía no tiene más función que la de enseñarnos de nuevo a verlas bien; y es verdad que la filosofía se realiza destruyéndose como filosofía separada". Phénoménologie de la perception, Paris, Gallimard, 1945, p. 520.
} 
filosofía y los sistemas filosóficos, tanto de Marx como de Kierkegaard, es una reacción contra el Hegel "escolarizado", no contra el Hegel de la Fenomenología del espíritu.

Merleau-Ponty diferencia dos períodos en la obra de Hegel. El primero, el de los escritos de juventud y el de la Fenomenología del espíritu; el segundo, el que va de la Lógica a la Enciclopedia. En el artículo "El existencialismo de Hegel", publicado en 1947, se advierte esta separación. En este texto, MerleauPonty, tras afirmar que "Hegel está en el origen de todo lo importante que se ha hecho en filosofía desde hace un siglo $[\ldots]^{\prime \prime}{ }^{9}$, planteaba el dilema acerca de si Hegel podía o no ser considerado un filósofo existencialista. La respuesta final será positiva, siempre y cuando nos acotemos a la Fenomenología del espíritu y no a los textos posteriores. Según Merleau-Ponty, mientras que el Hegel de la Lógica y de la Enciclopedia reduce la existencia a un momento del sistema, el Hegel de la Fenomenología del espíritu, por el contrario, intenta "[...] reencontrar el sentido de la historia total, [...] describir el movimiento interno de la substancia social, y no [...] explicar las aventuras de la humanidad a través de los debates de los filósofos" ${ }^{10}$. Para Merleau-Ponty, en el periodo de la Fenomenología del espíritu, Hegel parte del mundo concreto, mundo al cual quiere describir según su propio desenvolvimiento sin reducirlo a categorías ideales ni mucho menos. En la descripción de este desenvolvimiento Hegel toma la conciencia como algo no acabado. A diferencia de Kant, que partía de un sujeto universal, autónomo, puramente cognoscente, Hegel considera al hombre como algo que se está haciendo, que nunca está completo y que no es en forma alguna un mero sujeto del conocimiento. Por lo tanto, "[...] hay un existencialismo en Hegel en el sentido en que para él el hombre no es de entrada una conciencia que posee con toda claridad sus propios pensamientos, sino una vida dada a sí misma y que busca comprenderse"11.

De manera similar, en "Filosofía y no filosofía después de Hegel", MerleauPonty define al pensamiento que se despliega en la Fenomenología del espíritu como "a-filosofía", mientras que en su obra posterior ve una declinación de

\footnotetext{
9 Maurice Merleau-Ponty, Sens et non-sens, p. 109.

${ }^{10}$ Ibidem, p. 113.

${ }^{11}$ Maurice Merleau-Ponty, Notes des cours au Collège de France 1958-1959 et 1960-1961, p. 275.
} 
Hegel hacia la metafísica y la filosofía sistemática, donde la experiencia concreta se reduciría al concepto

\section{SABER Y ABSOLUTO}

A partir de la caracterización del pensamiento de Hegel como "a-filosofía", Merleau-Ponty aborda la relación entre el saber y lo absoluto desde la lectura de la Introducción a la Fenomenología del espíritu. Como dijimos en el punto anterior, la lectura que Merleau-Ponty lleva a cabo de Hegel se encuentra radicalmente alejada de las lecturas "escolarizadas". En el caso de la relación entre saber y absoluto, este alejamiento es explícito. Para Merleau-Ponty, en Hegel, ni lo absoluto es un principio metafísico ni el saber de lo absoluto puede ser reducido a un saber reflexivo.

En este sentido, Merleau-Ponty señala que, en Hegel, lo absoluto no debe ser comprendido como algo acabado, cerrado, ya constituido de una vez para siempre, ubicado en un mundo de orden superior sino que lo absoluto es el desenvolvimiento mismo del fenómeno, el cual acontece, en el "más acá" y no en el más allá"11. Merleau-Ponty señala que, al ser lo absoluto el desenvolvimiento del fenómeno, la vía de acceso a él no será otra que la fenomenología. Sin embargo, siguiendo el planteo de Hegel, Merleau-Ponty advierte que la fenomenología no debe ser pensada como un medio para conocer lo absoluto en el sentido de la problemática moderna en torno al método. "Principio poseído por Hegel: es por una fenomenología (aparición del espíritu) (espíritu del fenómeno) que se accede a lo absoluto. No porque el espíritu sea medio, instrumento, sino porque lo absoluto no sería absoluto si no apareciera así"12.

Merleau-Ponty tiene en cuenta la crítica explícita que Hegel realiza a la idea moderna de método. En las primeras líneas de la Introducción a la Fenomenología del espíritu, Hegel sostiene: "[...] en efecto, si el conocimiento es el instrumento para apoderarse de la esencia absoluta, inmediatamente se advierte que la aplicación de un instrumento a una cosa no deja a ésta tal y como ella es para sí, sino que la modela y altera"13. El planteo hegeliano se refiere al pro-

${ }^{12}$ Idem.

${ }^{13}$ G. W. F. Hegel, Fenomenología del espíritu, Buenos Aires, Fondo de Cultura Económica, 2007, p. 51. 
blema acerca de que el conocimiento, comprendido como medio o instrumento de acceso a lo absoluto, implica la imposibilidad de acceder a lo absoluto como tal, ya que sólo accederíamos a él a partir de la transformación que dicho medio ejerció sobre aquello a lo que deseamos acceder; lo que nos retrotraería a la problemática de la oposición kantiana entre el en sí y el fenómeno.

Para Merleau-Ponty, esta idea es central ya que pone de manifiesto el objetivo hegeliano de relativizar las oposiciones que suelen ser presentadas como antagónicas e irreductibles. Merleau-Ponty ve que, en Hegel, se da una superación de la concepción moderna que hace de la estructura triádica sujetométodo-objeto una unidad serial que une de manera externa tres elementos supuestamente independientes y autónomos. Precisamente, en la Fenomenología del espíritu, es la conciencia misma la que se hace saber de lo absoluto y los caminos por los que transita para llegar a esta instancia son también los propios caminos de lo absoluto. El sujeto deviene en el objeto sabido, a la vez que el objeto deviene el sujeto que sabe.

En este aspecto, según Merleau-Ponty, el pensamiento de Hegel se sustenta en dos postulados que se oponen a la mencionada división triádica: por un lado, la identidad de nuestro ser y del conocer; por otro, la idea de que pensar el saber es pensar una vida que se hace saber y en un saber que se hace vi$\mathrm{da}^{14}$. Merleau-Ponty aclara que, si bien, en un primer momento, estos dos postulados pueden parecer dogmáticos, en verdad no lo son. Por el contrario, ambos postulados significan el rechazo de todo tipo de dogmatismo ya que quiebran con las oposiciones surgidas del pensamiento reflexivo o, como suele decir Merleau-Ponty, de "sobrevuelo". Frente a las dualidades y oposiciones del pensamiento reflexivo, Merleau-Ponty reivindica de la fenomenología hegeliana el rol que ésta le otorga a la experiencia concreta del hombre en el mundo: "Fenomenología: esta autopresentación del espíritu, aparición que no es efecto de lo absoluto, sino lo absoluto mismo. De suerte que la filosofía es experiencia"15.

En este sentido, cabe mencionar el artículo de Jean-Nöel Cueille "La profundidad de lo negativo: Merleau-Ponty frente a la dialéctica hegeliana"16. En

${ }^{14}$ Maurice Merleau-Ponty, Notes des cours au Collège de France 1958-1959 et 1960-1961, p. 281.

${ }^{15}$ Ibidem, p. 282.

16 Jean-Noël Cueille, "La profondeur du négatif: Merleau-Ponty face à la dialectique de Hegel", en Renaud Barbaras / Mauro Carbone / Leonard Lawlor (eds.), Chiasmi International. Merleau-Ponty. De la Nature à l'Ontologie / From Nature to Ontology / Dalla Natura all'Ontologia, Paris / Milano / Memphis / Manchester, Vrin / Mimesis / University of Memphis / Clinamen Press, 2000. 
este texto se señala que Merleau-Ponty ve la fenomenología hegeliana como precursora de la fenomenología genética a la que él mismo adhiere. La fenomenología genética merleau-pontyana y la fenomenología hegeliana compartirían el hecho de pensar la ambigüedad del hombre con su entorno social y natural en un marco que no se agota en la perspectiva del conocimiento reflexivo sino que se sumerge en las profundidades de las estructuras ontológicas originarias sobre las cuales el conocimiento se funda.

Varios de los planteos de Merleau-Ponty, sirven de fundamento a la tesis de Cueille. En "Filosofía y no-filosofía después de Hegel", Merleau-Ponty relaciona el pensamiento de Hegel con un concepto característico de la fenomenología husserliana: el de "uno-en-el-otro" (Ineinander). "La conciencia, el verdadero progreso de un saber consiste, no en una comparación exterior de dos términos, sino en el Ineinander objeto-saber, noesis -noema que se entrecruzan $[\ldots]^{117}$. Precisamente, para el fenomenólogo francés, en Hegel la conciencia está ligada al objeto y su transformación conlleva la transformación del objeto, e inversamente. Esto le brinda a Merleau-Ponty la posibilidad de establecer una relación entre el pensamiento hegeliano, ciertos aspectos de la fenomenología genética husserliana, $y$, en particular, de su propia concepción de la fenomenología.

En este sentido, Merleau-Ponty reconoce en Hegel un esfuerzo por captar esos estratos profundos que anteceden a la emergencia de la conciencia reflexiva en toda su riqueza y complejidad. Merleau-Ponty revalida que, para Hegel, la conciencia reflexiva no sea el punto de partida metodológico. Las críticas merleau-pontyanas a la idea de un cogito translucido, en donde la identidad consigo mismo es el fundamento de toda la construcción del saber, encuentra en Hegel un predecesor. Con palabras de Merleau-Ponty, Hegel "inaugura la tentativa de exploración de lo irracional y de su incorporación en una razón ampliada que sigue siendo la tarea de nuestro siglo"18. La identificación del saber con la vida que Merleau-Ponty ve en Hegel significa un cambio de posición radical con respecto al paradigma de la reflexión. En este punto, Merleau-Ponty continúa manteniendo la misma tesis que en "El existencialismo de Hegel": el Saber Absoluto, último momento de la Fenomenología del espíritu, no es otra

\footnotetext{
17 Maurice Merleau-Ponty, Notes des tours au Collège de France 1958-1959 et 1960-1961, p. 301.

18 Maurice Merleau-Ponty, Sens et non-sens, p. 109.
} 
cosa que una vida sabiendo de sí misma. "Se intenta captar la relación del Erkennen con lo Absoluto como dada en nuestra vida (pues se trata de un Absoluto que será también Erkennen), de refundar los conceptos de subjetividad y objetividad, absoluto y conocimiento al contacto con nuestra vida [... $]^{19}$.

\section{ACTITUD CRÍTICA Y NEGATIVIDAD}

La comprensión de la Fenomenología del espíritu como "a-filosofía" y la consiguiente relativización de los postulados sobre los que se apoya el pensamiento de "sobrevuelo", le permiten a Merleau-Ponty contraponer la actitud crítica de la filosofía moderna con el pensamiento hegeliano. En un planteo que guarda un marcado paralelismo con el realizado por Lukács en Historia y conciencia de clase, Merleau-Ponty lleva a cabo una crítica de la actitud crítica ${ }^{20}$. En este sentido, Merleau-Ponty afirma que la actitud crítica se adjudica una radicalidad que en verdad no posee ya que se encuentra fundada en la separación entre sujeto y objeto y entre ser y saber. A partir de esta separación, MerleauPonty sostiene que la verdadera actitud radical es la de Hegel:

Esta actitud -la de Hegel- no es dogmática, sino, al contrario, la verdadera actitud crítica, radical; pues ella es la decisión de captar la forma en que se dan el concepto de lo absoluto, el Erkennen, lo objetivo, lo subjetivo (dieses Begriff zu geben), en lugar de presuponerlos como conocidos por todos o de dar como sobreentendidos los conceptos que de ellos se tiene ${ }^{21}$.

Para Merleau-Ponty, la actitud crítica parte de una diferenciación categórica entre el sujeto cognoscente, el objeto por conocer y el método como medio o instrumento por el cual es posible el acto de conocer. Es decir, la actitud crítica se sostiene en la estructura triádica a la que hicimos mención en el punto anterior, estructura ante la cual la crítica se detiene. Por el contrario, Hegel lleva a

\footnotetext{
19 Maurice Merleau-Ponty, Notes des cours au Collège de France 1958-1959 et 1960-1961, p. 282.

${ }^{20}$ En el primer capítulo de Historia y conciencia de clase, "¿Qué es el marxismo ortodoxo", Lukács sostiene que " [...] el punto de partida metódico de toda actitud crítica es precisamente la separación entre el método y la realidad, el pensamiento y el ser" (Lukács, Georg, Historia y conciencia de clase (vol. I), Orbis,Barcelona, 1985, p. 48.). Lukács realiza esta definición de la actitud y de la metodología críticas para contraponerlas a la dialéctica marxista, comprendida ésta como pensamiento de la totalidad. Precisamente, Lukács observa que el espíritu analítico, propio de la actitud crítica, se presenta a sí mismo como un progreso con respecto a la dimensión sintética-totalizadora de la dialéctica marxista. Sin embargo, para el filósofo húngaro, dicho progreso no es tal, ya que la dimensión analítica no puede ser otra cosa que un momento dentro del desarrollo del pensamiento dialéctico.

${ }^{21}$ Maurice Merleau-Ponty, Notes des cours au Collège de France 1958-1959 et 1960-1961, p. 282.
} 
cabo la verdadera crítica al cuestionar los fundamentos de la crítica misma. Según Merleau-Ponty, en la medida en que el pensamiento hegeliano sostiene que "nosotros somos en la verdad" 22 y que "el conocimiento de lo absoluto es contacto con lo absoluto" ${ }^{23}$, que sólo es tal en tanto se nos revela como una dimensión que nuestro propio ser pone al descubierto, se encamina hacia una dimensión más profunda que la de los postulados que fundamentan la crítica moderna. "Implicación de lo absoluto en nosotros como internos a la verdad, de un absoluto que no es otra cosa que la verdad de nosotros, que no está separada del Erkenen" ${ }^{24}$. La reciprocidad entre vida y saber implica un pensamiento mucho más originario que el de la separación que sirve de fundamento a la actitud crítica.

La exposición sobre la actitud crítica tiene, a su vez, como correlato la cuestión de la negatividad y el lugar que ésta ocupa en el movimiento hacia el saber. Si bien Merleau-Ponty reconoce que en la crítica moderna lo negativo cumple una función decisiva, la misma es muy distinta a aquella que posee en Hegel. Para Merleau-Ponty, la negatividad de la actitud crítica manifiesta sus limitaciones en tanto no afecta al sentido inicial de la verdad. Por el contrario, en la actitud crítica la negatividad sólo es un medio para reconstituir la verdad tal como aparece comprendida al comienzo del despliegue de la crítica. En Hegel, se trata, en cambio, de una negatividad que derrumba las nociones anteriores de verdad para llevar a ésta a un nivel de mayor complejidad.

En este sentido, Merleau-Ponty diferencia la duda cartesiana del escepticismo hegeliano. En Descartes la duda representa, según Merleau-Ponty, la restauración de la verdad bajo el mismo modo en que ésta se presentaba con anterioridad a la duda. Es decir, la duda es una negatividad que no tiene otra finalidad que reconstituir el sentido inicial de la verdad, que si bien puede modificar su contenido, no modifica su esencia. Por el contrario, el escepticismo hegeliano transforma no sólo los contenidos de la conciencia, sino la conciencia misma. Lo negado por el escepticismo no es únicamente una forma en la que la conciencia concibe el mundo, sino que es negada también la forma actual de la propia conciencia. En este aspecto, Merleau-Ponty sostiene que, tanto en la duda cartesiana como en la actitud crítica, la negatividad se mueve en los límites de la

\footnotetext{
22 Ibidem, p. 281.

${ }^{23}$ Ibidem, p. 283.

${ }^{24}$ Ibidem, p. 282.
} 
separación entre sujeto y objeto, la cual no sólo no es nunca negada sino ni siquiera puesta en duda. La negatividad hegeliana, en cambio, transforma la conciencia, su relación con el objeto, y el objeto mismo ${ }^{25}$. En este sentido, Merleau-Ponty señala que, en Hegel, el desenvolvimiento de la conciencia hacia la conciencia de sí por medio de la negatividad "[...] modifica al objeto mismo, deviene el segundo objeto, objeto sabido"26. Este segundo objeto "[...] que anula al primer objeto es la experiencia hecha sobre él $[\ldots]^{\prime 27}$.

Si bien Merleau-Ponty afirma el carácter negativo de la conciencia hegeliana, aclara que, no por ello, debe comprenderse la conciencia como "nada". "Esta conciencia que se forma o cultiva o se trabaja es una negación (puesto que lo inmediato es reconocido como no verdad), pero no es una negación que va al reino de la nada, sino que va a la nada de esto [...] de lo cual es resultado" ${ }^{28}$. La aclaración se enmarca en la prosecución del debate con Sartre, a quien menciona explícitamente. "[...] Solución de Sartre: sacrificio del para-sí, para que el en-sí sea, la nada es nada"29. Merleau-Ponty caracteriza al pensamiento de Sartre como un pensamiento extremo que se mueve en la alternativa radical entre el ser y la nada. Esta caracterización se encuentra ya presente en los escritos de los años cuarenta. En el artículo "La querella del existencialismo", Merleau-Ponty dice : "[...] el libro (El ser y la nada) es demasiado antitético: la antítesis de mi vista sobre mí mismo y del otro sobre mí, la antítesis del para-sí y del en-sí son a menudo presentados como alternativos, en lugar de ser descriptos como el lazo vivo del uno con el otro" ${ }^{30}$.

Si bien la tesis de Sartre se encuentra fundada, en parte, en una lectura de Hegel e incluso imbuida en un lenguaje marcadamente hegeliano, MerleauPonty se encarga de establecer la diferencia entre ambos autores. En Lo visible y lo invisible Merleau-Ponty se refiere a la filosofía de Sartre como una "filosofía de la negatividad absoluta". Para el autor de Signos, el planteo sartreano se mueve en un marco de oposiciones radicales que sólo pueden encontrarse en el pensamiento reflexivo ya que, en la existencia concreta, la relación entre la

\footnotetext{
${ }^{25}$ En Las aventuras de la dialéctica, Merleau-Ponty hace un planteo muy similar con respecto las diferencias entre Weber y Lukács. Según Merleau-Ponty, Lukács llevaría a cabo la relativización de la relación sujeto-objeto, que Weber nunca realizó. Esa sería la ventaja de los análisis de Lukács frente a los de Weber.

${ }_{26}$ Maurice Merleau-Ponty, Notes des cours au Collège de France 1958-1959 et 1960-1961, p. 292.

${ }^{27}$ Idem.

${ }^{28}$ Ibidem, p. 287.

${ }^{29}$ Ibidem, p. 300.

30 Maurice Merleau-Ponty, Sens et non-sens, p. 125.
} 
conciencia y el mundo, y entre el hombre y la naturaleza, exceden el ámbito de la negación. Merleau-Ponty sostiene que, en Hegel, se da una reciprocidad en donde las negaciones, al igual que las afirmaciones, son sólo parciales. En este aspecto, la lectura que realiza Merleau-Ponty de la negatividad en Hegel va en dirección de identificar la propia posición de una negatividad relativa con la del filósofo alemán. "Nada determinada y a un contenido, que no es pues Abgrund [...] sino pasaje (Übergang) a una nueva forma (neue Form) que es la verdad de la antigua, su verdadera superación" ${ }^{31}$.

\section{UNA DIALÉCTICA DE LA AMBIGÜEDAD}

La relativización de los términos antagónicos que Merleau-Ponty encuentra en Hegel significa, principalmente, una relativización de la oposición sujetoobjeto. Si tenemos en cuenta lo dicho por el propio Merleau-Ponty en Títulos y trabajos acerca de que uno de sus objetivos principales a lo largo de su obra ha sido intentar pensar una tercera dimensión donde el sujeto y el objeto se presenten como los reversos de un origen común, puede verse claramente la concordancia que el filósofo francés encuentra entre su pensamiento y el de Hegel. Merleau-Ponty observa que Hegel quiebra con los supuestos que delimitan de manera clara la diferencia entre la conciencia y el objeto: "[...] pues ella (la conciencia) es conciencia del objeto y de ella misma y del corte entre ellos $[\ldots]^{\prime \prime 32}$. La conciencia involucra al objeto, y se define a través del objeto, en tanto es conciencia de sí a partir del reconocimiento de su diferencia con el objeto. A su vez, por el lado del objeto, el objeto conocido deviene otro objeto, es decir, el saber del objeto significa el surgimiento de un nuevo objeto.

Esta interpenetración de los elementos opuestos que Merleau-Ponty ve en Hegel, le hace sostener que el pensamiento hegeliano es un pensamiento dialéctico en el sentido en que el propio Merleau-Ponty le da al término. La dialéctica hegeliana constituye, para Merleau-Ponty, un modo de pensar en el cual se diluyen las oposiciones radicales y en el que el devenir otro manifiesta la reversibilidad de términos a primera vista antagónicos. "La ambigüedad es

\footnotetext{
31 Maurice Merleau-Ponty, Notes des cours au Collège de France 1958-1959 et 1960-1961, p. 287.

32 Ibidem, p. 300.
} 
esencial a la dialéctica y al Erfahrung, pues es por ella que el objeto se convierte en sujeto e inversamente" ${ }^{\prime 33}$.

Jean-Nöel Cuelli, en el artículo ya mencionado, observa que Merleau-Ponty descubre una gran proximidad entre la dialéctica hegeliana y la idea de quiasmo. En efecto, en "Filosofía y no-filosofía después de Hegel", expresiones tales como bisagra (membrure), "reversibilidad" o uno-en-el-otro (Ineinander), son expresiones empleadas tanto para referirse a la dialéctica como para referirse al quiasmo. Ambas conceptos se entrelazan en un pensamiento de lo negativo que apunta al suelo común de la experiencia humana, donde el intercambio del ser y la vida se manifiestan de manera ambigua. En este sentido, Cuelli destaca que lo que Merleau-Ponty reivindica de la dialéctica hegeliana es su momento regresivo, rechazando el momento progresivo que implica el salto a lo especulativo. Si las coincidencias entre ambos autores se dan en ese "ir hacia atrás", en esa "regresión", en esa búsqueda de "profundidad" de las estructuras constituyentes de la experiencia humana, la divergencia se dará en la faceta "progresiva" en tanto Merleau-Ponty verá en ella un "elevamiento" sobre el mundo.

En varios trabajos, Merleau-Ponty define el pensamiento dialéctico como un modo de pensamiento alternativo tanto del pensamiento reflexivo como del pensamiento objetivo. Para el fenomenólogo francés, la dialéctica implica una relación intrínseca entre el pensamiento y la historia que coloca a la filosofía en una dimensión que trastoca las concepciones tradicionales. En Lo visible y lo invisible, Merleau-Ponty, afirma que, a diferencia del pensamiento reflexivo que intenta legitimar el mundo a partir de las operaciones de la conciencia, el pensamiento dialéctico parte del supuesto de que "[...] hay ser, hay mundo, hay algo; hay cohesión, hay sentido $[\ldots]^{\prime \prime 34}$. La dialéctica toma, por tanto, como punto de partida lo dado, y en ningún momento se postula a sí misma como justificación del mundo, ni mucho menos como instancia constitutiva de éste. Merleau-Ponty habla de la dialéctica como de una "[...] fijación secundaria de una experiencia anterior $[\ldots]^{\prime 35}$. La dialéctica describe el mundo y no intenta legitimarlo a partir de conceptos intelectuales.

Regresando al debate con Sartre, en su libro De l'être du phénomène, Renaud Barbaras sostiene que una de las principales diferencias entre la filosofía

\footnotetext{
${ }^{33}$ Maurice Merleau-Ponty, Notes des cours au Collège de France, p. 301.

${ }^{34}$ Merleau-Ponty, Maurice, Le visible et I'invisible, p. 115.

35 Ibidem.
} 
de Sartre y la de Merleau-Ponty es la manera en que cada uno concibe la dialéctica ${ }^{36}$. Barbaras señala que mientras la dialéctica sartreana es una dialéctica de antinomias irreconciliables, la merleau-pontyana representa una acción recíproca entre las partes, un entrelazamiento en el cual un elemento lleva necesariamente al elemento que niega y viceversa. Precisamente, Merleau-Ponty ve el pensamiento de Sartre como una filosofía de la "negatividad absoluta", un pensamiento no-dialéctico, o, en todo caso, un exponente de la "mala dialéctica". Merleau-Ponty sostiene que la filosofía dialéctica no habla de una irrupción originaria de la nada en la plenitud del ser, puesto que se sabe dentro de un mundo donde hay ser y donde hay también nada; pero esta nada no es comprendida en sentido absoluto. Hay apariencias que mutan, y esta mutación implica un volverse nada de la apariencia anterior; sin embargo, eso que desaparece, que se vuelve nada, es reemplazado por una apariencia nueva. El ser y la nada se entrelazan siempre, a cada instante, están siempre ahí, pero su sentido es relativo y no absoluto. La dialéctica piensa y describe ese entrelazamiento, no como abstracciones ya prefijadas sino como movimiento siempre relativo.

En el resumen de curso titulado "La filosofía dialéctica", Merleau-Ponty enumera tres elementos que constituyen el pensamiento verdaderamente dialéctico. Por un lado, se trata de un pensamiento de los contradictorios pero que no busca anular la oposición sino que mantiene siempre la tensión. Por otro, es un pensamiento que no toma al ser en sí de manera objetiva ya que establece un nuevo sentido de la relación entre la subjetividad y el mundo. Finalmente, lo propio del pensamiento dialéctico es ser un pensamiento circu$\operatorname{lar}^{37}$.

Merleau-ponty advierte sobre la existencia de una "mala dialéctica", la cual se impone el objetivo de amoldar la realidad a un modelo formal preestablecido. Es decir, esta "mala dialéctica" estaría constituida como un principio formal del pensamiento y forzaría a la realidad a adecuarse a ella. Tesis, antítesis y síntesis, serían momentos ineludibles de lo real y todo lo que "es" debería ser pensado bajo este criterio. "Nos hallamos ya en la vía de la mala dialéctica, de la que, en contra de sus principios, impone una ley y un marco exteriores al contenido y restaura el beneficio propio del pensamiento pre-dialéctico" ${ }^{38}$.

\footnotetext{
${ }^{36}$ Renaud Barbaras, De l'être du phénomène, Paris, J. Millon, 1991, p. 135.

37 Maurice Merleau-Ponty, Résumés de cours. Collège de France 1952-1960, pp. 78-81.

38 Ibidem, p. 123.
} 
Sin lugar a dudas, Merleau-Ponty está pensando, principalmente, en la concepción de la dialéctica utilizada por el marxismo de la línea pro-soviética. En efecto, la teoría materialista del estalinismo comprende a la dialéctica de manera abstracta. El desarrollo de lo real es pensado como el desarrollo de la materia a través de etapas de tesis, antítesis y síntesis. A partir de este modelo se explica la totalidad de lo existente sin tomar en cuenta las particularidades históricas. No se piensa, pues, el mundo, sino que lo que se piensa es la manera de "acomodar" lo existente a la teoría pautada de antemano. Esta dialéctica, a su vez, instituye una necesidad de tipo causal que hace que todo el movimiento esté determinado, por lo cual la historia tendría un fin ineludible, en este caso la Revolución comunista.

Esta forma de comprender la dialéctica es, para Merleau-Ponty, una extensión del pensamiento objetivista que se encuentra en las antípodas de la apuesta merleau-pontyana. Se trata de un pensamiento de "sobrevuelo" que no se sitúa en el mundo ni en la experiencia concreta, y donde la riqueza y complejidad del mundo y de la historia, no sólo es ignorada sino también aplastada por un modelo intelectual que se presenta como lo único verdadero. En "Filosofía y no-filosofía después de Hegel", la dialéctica hegeliana no tiene nada que ver con esta "mala dialéctica"; por el contrario, se trata de un pensamiento que intenta comprender la pluralidad del mundo, el enriquecimiento mutuo de los elementos opuestos y la reciprocidad de las relaciones.

\section{CONCLUSIÓN}

Según nuestra perspectiva, el abordaje de temas como los hasta aquí expuestos tiene un doble interés. Por un lado, puede servir para enriquecer el estudio y la comprensión del pensamiento merleau-pontyano. A veces ocurre que las lecturas que Merleau-Ponty realiza sobre Hegel son pasadas por alto, lo mismo que sus lecturas sobre Marx. Sin embargo, tanto en el caso de Hegel como en el caso de Marx, se trata de pensadores que siempre estuvieron presentes en el desarrollo del pensamiento y de la obra de Merleau-Ponty, o bien como fines en sí mismos o bien como medios para comprender otras corrientes o, incluso, acontecimientos históricos o sociales. 
Por otra parte, la forma en que Merleau-Ponty se enfrenta a la obra de Hegel, puede servir también para arrojar nuevas perspectivas acerca de la filosofía hegeliana. En este sentido, una característica muy propia de MerleauPonty es, a nuestro parecer, la de ser un gran lector de otros filósofos. La comprensión del pensamiento de Hegel como a-filosofía, como un pensamiento que trasciende los sistemas y que se abre a lo absoluto por medio de una experiencia que excede el ámbito de la pura reflexión, es muy interesante para contraponerla al Hegel "escolarizado" denunciado por el propio Merleau-Ponty.

Por último, resulta también interesante e invita a la polémica su tesis acerca de los dos momentos que atravesaría la filosofía de Hegel. En este punto, Merleau-Ponty sostiene con respecto a Hegel la misma tesis que con Marx: habría un primer momento (en el caso de Hegel, Fenomenología del espíritu y las obras anteriores a 1807, y, en el caso de Marx, los escritos de juventud) en el que no se intenta apresar la experiencia por medio del concepto y reducir a él las manifestaciones y expresiones de la historia humana; y habría un segundo momento (el de la Lógica y la Enciclopedia en Hegel y el de El Capital en Marx) en el que ambos autores recaerían en concepciones metafísicas a partir de las cuales se intentaría apresar las ambigüedades y complejidades de la experiencia concreta en un sistema filosófico. "Notoriamente, el pasaje del joven Marx al Capital no será tanto el pasaje de la filosofía a la ciencia como el pasaje de la Fenomenología a la Lógica" ${ }^{39}$.

${ }^{39}$ Maurice Merleau-Ponty, Notes des cours au Collège de France, p. 325. 